\title{
Disruptionen in der Schule aus der Sicht von Lehrer*innen
}

\author{
Carlo Schmidt
}

\section{Zusammenfassung des Beitrags}

Welche Sicht haben Lehrer*innen an zwei ausgesuchten Schulen auf den Notfall-Fernunterricht, der aufgrund der politischen Maßnahmen während der Corona-Pandemie nötig wurde? Mithilfe von Diskursiven Interviews wurden Lehrer*innen befragt, die tagtäglich mit den entstehenden Herausforderungen konfrontiert sind. Das Ziel des Beitrags ist es, die sozialen Deutungsmuster der Lehrer*innen im Zusammenhang mit eben diesen Herausforderungen zu rekonstruieren. Im Ergebnis zeigte sich, dass dabei besonders die Frage der digitalen Reproduzierbarkeit des Sozialen sowie pragmatische Muster die Deutungen der Lehrer*innen bestimmen.

Schlüsselbegriffe: Lebrer*innen • Notfall-Fernunterricht • Deutungsmusteranalyse • Diskursives Interview

\section{Einleitung und Relevanz}

Die Organisation von Schule und Unterricht in den Schuljahren 19/20 und 20/21 war besonders durch die COVID-19 induzierten politischen Maßnahmen geprägt. Eine solche Disruption eines bis dato institutionell und organisatorisch stabilen Prozessmusters erfordert allgemeinhin eine Neuformierung der Organisation und der Ordnung innerhalb des Systems Schule. Schließlich werden die alltäglich ablaufenden Prozessmuster durch die Struktur und die Logik des Systems hervorgebracht. Besonders beachtenswert ist dabei, dass sich durch den Handlungsdruck der Wechsel der Prozessmuster relativ schnell vollziehen musste, wobei jedoch die Struktur und die Logik des Systems weitgehend gleichblieb. Das Umschalten auf den Notfall-Fernunterricht - und dieser dann selbst - war somit ein neues Prozessmuster, das sich mittels größtenteils gleichbleibender Logik des Systems vollziehen musste.

Die Disruptionen, die in den Schulen stattgefunden haben und durch die der Notfall-Fernunterricht notwendig wurde, werden schon allein deshalb zu einem medienpädagogischen Thema, da der Fernunterricht vor allem erst durch digitale Medien ${ }^{1}$ möglich gemacht wird.

\footnotetext{
${ }^{1}$ Hier kann Marshall McLuhans Stehsatz „Das Medium ist Botschaft“ (vgl. 2016) bemüht werden. Die Botschaft der Medien, in Form von digitalen Lernangeboten/-plattformen, ist in diesem Fall die Möglichkeit des Notfall-Fernunterrichts.
} 
Diese These sei damit gestützt, dass Unterricht eine soziale Dimension mit sich bringt, die wenn auch nur in marginalen Ansätzen - im Notfall-Fernunterricht durch Medien realisierbar ist (siehe 4.). Die hier rekonstruierten Deutungsmuster der Lehrkräfte sind deshalb medienpädagogisch relevant, da sie aus einer Zeit rekonstruiert wurden, in der Unterricht größtenteils (stellenweise ausschließlich) nur durch digitale Medien möglich war; der Begriff „Medienpädagogik" also dahingehend Bedeutung erlangt, dass in der Zeit der Schulschließungen und Kontaktverbote „Pädagogik“ in den Schulen vor allem durch und mit (digitalen) Medien ${ }^{2}$ möglich war.

Seit Beginn der Corona-Pandemie erschienen zahlreiche erziehungs- und bildungswissenschaftliche Auseinandersetzungen mit den Auswirkungen der politischen Maßnahmen aufgrund des Infektionsgeschehens auf das Bildungssystem. Einige seien hier exemplarisch genannt: Fickermann und Edelstein (vgl. 2020) stellen einerseits die Herausforderungen dar, die durch den Fernunterricht entstehen und andererseits die schulischen Handlungsfelder während und nach der Pandemie. Im Schulbarometer von Huber et al. (vgl. 2020a) werden Themen wie beispielsweise Kommunikation, Fernunterricht, Belastung, Bedarfe und Zusammenarbeit in den Schulen quantitativ erfragt. Drerup und Schweiger (vgl. 2020) bieten philosophische Reflexionsangebote in Form kurzer Essays. Helm/Huber/Loisinger (vgl. 2021) geben darüber hinaus in ihrem umfassenden Review einen systematischen Überblick über 97 quantitative Studien. In eine etwas andere Richtung geht die vom VBE beauftrage Befragung von 260 Schulleiter*innen in Baden-Württemberg zu deren Sicht der Schule vor und während der Corona-Krise (vgl. forsa 2020) ${ }^{3}$. Diese regionale Perspektive erscheint besonders aufgrund des Föderalismus und der Regionalisierungsinitiativen (Hamborg 2018; Niedlich 2020) im deutschen Bildungssystem zielführend. Auffallend in all diesen Publikationen ist, dass Lehrer*innen (außer bei Huber et al. 2020a und Helm/Huber/Loisinger 2021 an einigen Stellen, sowie VBE 2021) nicht zu Wort kommen.

Mit dieser Studie soll dazu beigetragen werden, diese Lücke zu schließen, indem erstens Lehrer*innen zu Wort kommen, die für das tagtäglich veranstaltete (Notfall-Fern-)Unterrichtsangebot (vgl. Fend 2008, S. 120) sorgen. Zweitens wird, um den Eigenheiten des Föderalismus gerecht zu werden, regional perspektivisch vorgegangen. Dazu wurden zehn Lehrkräfte aus zwei SEK-I Schulen im Großraum Stuttgart interviewt ${ }^{4}$. Methodisch wird, drittens, ein qualitativ-rekonstruktiver Ansatz mittels des Diskursiven Interviews (vgl. Ullrich 2020) gewählt.

\footnotetext{
${ }^{2}$ Begriffsanalytisch kann gefragt werden, ob Pädagogik überhaupt ohne Medien möglich ist. Eine Antwort darauf ist abhängig von der Weite der Begriffsfassung, da sehr vielfältige Entitäten als Medien verstanden werden können. Zur Verdeutlichung ein kurzes Beispiel: Es kann von der Pädagogik der Medien (beispielsweise dem erzieherischen Wert von Tabletts, Zeitungen, etc.) oder diametral von den Medien der Pädagogik (beispielsweise Kinder, vgl. hierzu Luhmann 1991) gesprochen werden. Beide Male wird deutlich, dass Medien für den theoretischen Fluchtpunkt bedeutsam sind, jedoch etwas gänzlich anderes darunter verstanden wird. In Abgrenzung zu anderen Disziplinen der Erziehungswissenschaft und in Bezug zum Thema des Beitrages (dem Fernunterricht aufgrund von Schulschließungen) sei hier deshalb bestimmt, dass mit dem Begriff „Medien“ die Beschäftigung mit der Bedeutung von digitalen Medien, also Computern, Tablets, Apps und des Internets für die Pädagogik gemeint ist.

${ }^{3} \mathrm{Zu}$ bemerken ist dabei, dass die Schulleitungen ihre Einschätzungen bereits im Mai 2020 abgegeben haben.

${ }^{4}$ Eine ausführlichere Beschreibung der Samplingstrategie findet sich in Abschnitt 3 dieses Beitrages.
} 
Das Ziel ist dabei die Rekonstruktion der Deutungsmuster, die Lehrer*innen (hier aus dem Großraum Stuttgart) im Zusammenhang mit den Disruptionen aufzeigen. Der vorliegende Beitrag versucht damit durch diesen Ausschnitt, einen Teil zu einer differenzierteren Gesamtperspektive zu leisten.

Nachdem zunächst der theoretische Kontext (1.) dieses Beitrages dargelegt wird, werden die Forschungsfragen (2.) formuliert. Daraufhin werden die Ausführungen zur Methodologie, Methodik und Durchführung (3.) der Studie dargestellt. Es folgen die Analyse und Rekonstruktion der Deutungsmuster der Lehrkräfte (4.). Der Beitrag schließt mit einer Diskussion der Erkenntnisse (5.).

\section{Theoretischer Kontext}

Da der Begriff des Deutungsmusters stellenweise unterschiedlich und oft in eher loser Form Verwendung findet (vgl. Ullrich 2020, S. 3; Meuser 2006, S. 31) und damit keine kanonisierte Begriffsbestimmung vorhanden ist, auf die zurückgegriffen werden könnte, wird im Folgenden kurz dargelegt, was in dieser Studie unter dem Deutungsmusterbegriff verstanden wird und wie dieser aus einem diskursiven Verständnis herleitbar ist.

Die Diskussionen um den Begriff „Deutungsmuster“ begannen im deutschen Sprachraum im Jahr 1973, mit einem Text von Ulrich Oevermann (vgl. 1973). Dort wird ausgeführt, dass soziale Deutungsmuster ihre eigene (Argumentations-)Logik sowie eigene Regeln der Vernünftigkeit und Gültigkeit aufweisen (vgl. Oevermann 1973, S. 3). In Anlehnung an Lüders und Meusers (1997) Unterscheidung zwischen einer strukturtheoretischen und einer wissenssoziologischen Perspektive auf Deutungsmuster - in der sie Oevermann der strukturtheoretischen Perspektive zuordnen - verfolgt dieser Beitrag eine wissenssoziologische Perspektive. Im Gegensatz zur strukturtheoretischen Perspektive „wird dabei die generierende und gestaltende Rolle handlungsfähiger Subjekte betont" (ebd., S. 62; vgl. hierzu auch der nächste Absatz dieses theoretischen Teils). Begriffshistorisch wurden ausgehend von Oevermanns initialer und umfangreicher Auseinandersetzung mit dem Deutungsmuster unterschiedliche Versuche unternommen, eine Begriffsbestimmung zu leisten. Durch die Jahre hinweg ist dabei eine begriffliche Schnittmenge feststellbar: Deutungsmuster dienen Akteur*innen als ein Orientierungsund Rechtfertigungspotential für die Bewältigung von alltäglichen Handlungsproblemen (vgl. Arnold 1983, S. 894; Oevermann 2001, S. 539; Keller 2014, S. 146). Dabei werden Deutungsmuster „als kollektive Produkte begriffen, die im gesellschaftlichen Wissensvorrat vorhanden sind und sich in konkreten sprachlichen Äußerungen manifestieren“ und weiter organisieren Deutungsmuster ,individuelle bzw. kollektive Erfahrungen und sie implizieren meist Vorstellungen (Modelle) angemessen Handelns" (Keller 2011a, S. 240)5. Auch Ullrich (2020, S. 14) stellt fest, dass soziale Deutungsmuster Teil des kollektiven Wissensvorrats sind und merkt zusätzlich an, dass Deutungsmuster grundsätzlich miteinander konkurrieren sowie im Widerspruch zueinander stehen können. Es muss demnach eine trennscharfe Unterscheidung

\footnotetext{
${ }^{5}$ Zur Idee des „gesellschaftlichen Wissensvorrates“ siehe auch Lüders und Meuser (1997, S. 64).
} 
zwischen einem sozialen und einem individuellen Deutungsmuster vorgenommen und im gleichen Atemzug deren Zusammenhang betont werden. Dieser trennscharfe Zusammenhang lässt sich auf den genuinen Ort der Deutungsmuster zurückführen - die Diskurse.

Im Anschluss an Foucault werden Diskurse hier verstanden als „regulierte Produktionsprozesse von Aussagesystemen, die geltendes Wissen über die Wirklichkeit herstellen und insofern $>$ Wahrheit< legitimieren - soziale Denksysteme also, in denen bestimmte Vorstellungen konsensuell festgeschrieben sind" (Schetsche/Schmied-Knittel 2013, S. 26). Dieser Logik folgend formieren Diskurse Subjekte zwar, jedoch werden Diskurse auch durch Subjekte hervorgebracht, wodurch eine gleichzeitige Hervorbringung von und Unterwerfung unter Diskurse stattfindet (vgl. Foucault 2016, S. 37) ${ }^{6}$. Die Subjekte sind damit gleichsam Produzent und Produkt von Diskursen. Die Produktionsmittel sind dabei (unter anderem) die Deutungsmuster - die ihrerseits zunächst auch produziert werden müssen. Somit gilt hier für Deutungsmuster das Gleiche wie für Diskurse: auch Deutungsmuster werden durch Subjekte produziert und produzieren dann wiederum Subjekte. Folglich können Deutungsmuster zugleich als handlungsermöglichend und handlungssteuernd beschrieben werden (vgl. Ullrich 2020, S. 11, H. i. O.). Daran kann angefügt werden, dass Diskurse und Subjekte damit sogleich Normalismen (vgl. Link 2006) produzieren, die dann das Werte- und Wahrnehmungssystem der Subjekte mitgestalten. Angemerkt sei an dieser Stelle, dass zwischen Diskursen - und damit auch zwischen Sprache - und Gesellschaft kein bloßer Determinismus besteht (vgl. Jäger 2012, S. 24); Diskurse und damit auch Sprache jedoch als „transsubjektive Produzenten gesellschaftlicher Wirklichkeiten und sozio-kultureller Deutungsmuster gefasst werden können “ (Jäger 2012, S. 28).

In diesem Sinne handelt es sich beim Deutungsmusterkonzept um ein „Brückenkonzept“, das es erlaubt, den Weg von der Diskursebene zur alltäglichen Deutungs- und Lebenspraxis zu gehen und so die unterschiedlichen Begründungen in den Blick zu nehmen (vgl. Keller 2014, S. 155). Diese „unterschiedlichen Begründungen“ werden hier als individuelle Derivationen ${ }^{7}$ verstanden. Im vorliegenden Verständnis werden soziale Deutungsmuster demnach durch ihre Anwendung als Derivation immer wieder bestätigt, reproduziert und verändert. Dabei wird deutlich, „dass dem Begriff des Deutungsmusters als Analysekategorie die zentrale, sondierende Stellung zukommt, zwischen einem real existenten gesellschaftlichen Wissensvorrat und den individuellen Handlungen eines Individuums zu vermitteln“ (Brandmayr 2018, S. 23).

Zusammenfassend wird damit zur Schärfung der Perspektive der Analyse zwischen individuellen Deutungsmustern, die das situative Handeln orientieren und Derivationen, also denjenigen Deutungsmustern, die zur Kommunikation und Legitimation der Handlungen

\footnotetext{
${ }^{6}$ Ganz ähnlich argumentieren Schetsche und Schmied-Knittel (2013, S. 25): „Damit stehen soziale Deutungsmuster und Diskurse in einem wechselseitigen Beziehungs- und Bedingungsgeflecht auf der Mikro- und Makroebene: Individuelle Handlungen müssen nicht nur mit sozial geteilten Deutungsmustern kompatibel sein (um verstanden zu werden), sondern jene primär lebensweltlich relevante Wissensform unterliegt ihrerseits diskursiven Mechanismen“.

${ }^{7}$ Der in dieser Studie verwendete Derivationsbegriff bezeichnet „die in Handlungsbegründungen verwendeten Deutungsmuster" (vgl. Ullrich 2020, S. 14).
} 
herangezogen werden, unterschieden (vgl. Ullrich 2020, S. 14), um schließlich soziale Deutungsmuster, als Teil eines kollektiven Wissensvorrats der interviewten Lehrkräfte zu rekonstruieren.

\section{Forschungsfragen}

Aus der Verbindung der dargestellten Effekte der Schulschließungen (Einleitung und Relevanz) und der theoretischen Annahmen (1.) ergeben sich die im Folgenden aufgeführten Forschungsfragen. Diese Forschungsfragen wurden im Leitfadeninterview (3.) als Hauptfragen verarbeitet und werden deshalb kurz dargestellt.

Erstens interessiert, wie die Lehrer*innen mit den Herausforderungen, die der Notfall-Fernunterricht mit sich bringt, umgangen sind. Zum einen wurde mit der Frage: „Welche mediale Ausstattung ist an Ibrer Schule vorbanden?" die Beleuchtung der institutionellen Grundlagen und Herausforderungen des Unterrichts eingeleitet. Zum anderen zielte die Frage: „Auf welche sozio-ökonomischen Herausforderungen sind Sie während der Zeit der Schulschließungen gestoßen?" in Richtung der Herausforderungen in Form von Chancengleicht und der Bildungsbenachteiligung aufgrund der Schulschließungen. Dieses Themenfeld findet sich bei Huber und Helm (vgl. 2020b), Wrase (vgl. 2020), Klieme (vgl. 2020), Köller et al. (vgl. 2020), Goldan/Geist/Lütje-Klose (vgl. 2020) sowie Bremm und Racherbäumer (vgl. 2020) wieder, in Verbindung mit vulnerablen Gruppen auch bei Schweiger (vgl. 2020).

Zweitens wird im Sinne der Suche nach Möglichkeitsbedingungen fokussiert, welche Potentiale die Lehrer*innen im Einsatz von digitalen Lernangeboten während und nach der Pandemie sehen. Die Frage an die Teilnehmer*innen war in diesem Fall: „Welche Potentiale seben Sie im Einsatz von digitalen Lernangeboten - wäbrend der Pandemie und darüber hinaus?"Diese Frage wird vor allem aufgrund der starken Polarisierung von digitalen Lernangeboten im breiten öffentlichen Diskurs gestellt. Für den wissenschaftlichen Diskurs sei hier exemplarisch auf der einen Seite auf Tenorth (vgl. 2020) ${ }^{8}$ als eher kritische Stimme und auf der anderen Seite auf die fachdidaktischen Beschäftigungen mit dem Einsatz von digitalen Medien im Unterricht (vgl. Maurer/Rincke/Hemmer 2021) hingewiesen.

Drittens konzentriert sich die Aufmerksamkeit darauf, was von den befragten Lehrer*innen als notwendig erachtet wird, um generell zielführende digitale Bildungsangebote an den Schulen anbieten zu können. Dazu wurden die Teilnehmer*innen zu einem Gedankenexperiment aufgefordert: „Stellen Sie sich vor, Sie müssten die Schulen für einen guten Fernunterricht fit machen. Was würden Sie tun?"Der Frage geht die Auseinandersetzung mit dem Fernunterricht als Problemfeld voraus (vgl. Porsch/Porsch 2020, Wacker/Unger/Rey 2020, Wrase 2020, Klieme 2020, Köller et al. 2020 sowie Gogolin 2020) und geht von der Heuristik aus, dass

\footnotetext{
${ }^{8}$ Hier soll betont sein, dass sich Tenorth nicht grundlegend „dagegen“ ausspricht, sondern die Stimmung „Hurra! Digitalisierung!“ kritisch betrachtet. Im Vergleich hierzu: soziale Deutungsmuster der interviewten Lehrkräfte (4. und 5.).
} 
Lehrkräfte die Experten des (eigenen) Unterrichtens sind, die in jeder digitalen Unterrichtsstunde feststellen, was funktioniert und was nicht.

Viertens wurde ausgeleuchtet, welchen Stellenwert die Teilnehmer*innen der Digitalisierung in der Bildung zuschreiben. Gefragt wurde dabei: „Welchen Stellenwert hat Ibrer Meinung nach „die Digitalisierung “für eine gelungene Bildung von Schüler*innen?" Da die Gesellschaft digitale Muster aufweist (vgl. Nassehi 2019) und „Digitalisierung“ eine Omnipräsenz in weiten Teilen des öffentlichen schulbezogenen Diskurses beansprucht, interessiert, wie Lehrer*innen im Zusammenhang mit „Bildung“ darüber denken. Im jüngeren, pandemiebezogenen Schuldiskurs findet sich die Auseinandersetzung mit Digitalisierung und Schule bei Huber und Helm (vgl. 2020b), Hoffmann (vgl. 2020), Wrase (vgl. 2020) und Fischer/FischerOntrup/Schuster (vgl. 2020).

Die Detailfragen wurden situativ gestellt und sind deshalb hier nicht immer einer bestimmten Hauptfrage zuordenbar. Das ist der Interviewführung geschuldet: Die Lehrer*innen wurden in ihrem Erzählfluss nicht unterbrochen und haben so stellenweise eigenständig Themen angeschnitten, auf die dann eingegangen wurde.

\section{Methodologie, Methodik, Durchführung}

Für die vorliegende Studie zentral ist, dass „soziale Wissensformen nicht ausschließlich auf kollektiver Ebene analysiert werden können - schon allein deshalb nicht, weil gesellschaftliche Wissensvorräte nun einmal im lebensweltlichen Handeln einzelner Akteure praxiswirksam werden" (Schetsche/Schmied-Knittel 2013, S. 25, H. i. O.). Soziales Wissen, tragen die Akteure demnach in sich und gestalten damit ihren Alltag. Die im Folgenden eingeschlagene methodische Richtung setzt das Verständnis voraus, dass „[d]ie objektivierte soziale Welt von der Sprache auf logische Fundamente gestellt [wird]. Das Gebäude unserer Legitimationen ruht [demnach, CS] auf der Sprache, und Sprache ist ihr Hauptargument." (Berger/Luckmann 2013, S. 69). Damit einher geht dann auch, dass die Wahrnehmung der sozialen Welt und damit auch die soziale Welt selbst, zu großen Teilen das performative Produkt der Begriffe [sein muss], durch die wir auf sie blicken (vgl. Eribon 2017, S. 122). Gesellschaftliche Wirklichkeit wird demnach durch unsere Sprache mit-konstituiert (vgl. Rosa 2021, S. 9).

Die mit den Lehrkräften geführten Interviews orientieren sich an der Methode des Diskursiven Interviews (vgl. Ullrich 2020), das „zur Erfassung und Rekonstruktion sozialer Deutungsmuster" (Ullrich 2020, S. 1) entwickelt wurde. Das Diskursive Interview erscheint deshalb zielführend, da es sich durch drei spezifische methodologische Prämissen auszeichnet (vgl. Ullrich 2020, S. 38 ff.):

Erstens liegt ein wissenssoziologisches Grundverständnis vor. Dabei muss zwischen zwei Linien der deutschen Wissenssoziologie unterschieden werden. Zum einen die Linie von Max Scheler und Karl Mannheim, die sich für elaborierte Wissensformen, also beispielsweise für Ideologien und/oder wissenschaftliches Wissen interessieren (vgl. Kaldewey 2014, S. 4). Zum anderen die Linie von Berger und Luckmann, die sich in den 1960er-Jahren dezidiert von der 
theoretischen Perspektive der Intellektuellen (also von Scheler und Mannheim) abwenden und stattdessen das vortheoretische Wissen des alltäglichen Lebens in den Fokus rücken (vgl. Kaldewey 2014, S. 4, H. i. O.). Diese Wissensform wird im Paradigma von Berger und Luckmann als das „Allerweltwissen“ bezeichnet und bildet dort die Bedeutungs- und Sinnstruktur einer Gesellschaft (vgl. Berger/Luckmann 2013, S. 16). Zurückzuführen ist dieses wissenssoziologische Verständnis von Wissen auf die phänomenologische Perspektive von Alfred Schütz (vgl. Kaldewey 2014, S. 4), der das von jedermann als selbstverständlich hingenommene Wissen zum Ausganspunkt seiner Analysen nimmt (vgl. Schütz 1971, S. 86). Da davon auszugehen ist, dass Lehrkräfte einmal im Studium erlernte Wissensbestände eigenständig-konstruktiv verwenden und an der Praxis abarbeiten (vgl. Kolbe/Combe 2008) - so also wiederum aus der Perspektive der Lehrkräfte selbstverständliches Wissen produzieren - wird an dieser Stelle in der Linie von Berger und Luckmann weitergearbeitet.

Zweitens wird davon ausgegangen, dass Deutungsmuster in Gesprächen - in diesem Fall in einem Interview - kommuniziert und zur Begründung von Handlungen verwendet werden. Woran sich drittens anschließt, dass soziale Deutungsmuster vergleichend rekonstruiert werden können. In Abgrenzung zu anderen Formen qualitativer Interviews ist zu betonen, dass das Diskursive Interview explizite Fragetechniken und eine entsprechende Interviewführung vorsieht (vgl. Ullrich 2020, S. 54): Die Interviewenden verlassen die Position des passiven Fragenstellens. Stattdessen wirken sie aktivierend in die Interviewsituation ein, indem durch unterschiedliche Arten von Sprech- und Begründungsaufforderungen sehr bewusst und notwendig am Prozess der Hervorlockung von Derivationen mitgewirkt wird (vgl. Ullrich 2020, S. 54). Gerade dieses „Eingreifen“ grenzt das Diskursive Interview zu anderen qualitativen Methoden ab. Für das Diskursive Interview sind Begründungsaufforderungen jedoch von großer Bedeutung, da davon ausgegangen wird, dass in diesen Begründungen soziale Deutungsmuster als Derivationen zum Vorschein kommen (vgl. Ullrich 2020, S. 91). Um zu verhindern, dass sich die Begründungsaufforderungen negativ auf die Interviewsituation auswirken, wurde im Vorfeld auf das Auftreten etwaiger Begründungsaufforderungen oder dergleichen aufmerksam gemacht. Dabei ist davon auszugehen, dass nicht jedes Mitglied eines Deutungsmusterkollektivs - hier: die einzelne Lehrkraft - alle verfügbaren Deutungsmuster kennt (vgl. Ullrich 2020, S. 14). Deshalb werden durch das einzelne Interview sowohl die vom einzelnen Subjekt verwendeten Derivationen als auch die individuellen Deutungsmuster ersichtlich. Dabei entstehen demnach zunächst Einzelfälle.

Das hat zur Folge, dass die Interviews in Bezug zueinander gestellt werden müssen, da soziale Deutungsmuster „nur durch den Vergleich, und das ,Übereinanderlegen““ (Ullrich 1999, S. 443, H.i.O.) von Einzelfällen identifizierbar und rekonstruierbar sind. Um dem gerecht zu werden, wurde bei der Analyse der Interviews queranalytisch kontrastiert, wodurch „[d]ie Bildung von Idealtypen [...] aufgrund empirisch vorgefundener Merkmale“(Ullrich 1999, S. 444) vorgenommen wurde. Zur Vorbereitung der Queranalyse wurde innerhalb der Interviews mittels minimaler und maximaler Kontrastierung codiert (vgl. Keller 2011b, S. 113 f.; Ullrich 2020, S. 131 f.). Methodisch ist der Codierungsprozess dabei an Aspekten der Grounded 
Theory (vgl. Breuer/Muckel/Dieris 2019; Strauss/Corbin 1996) angelehnt, wobei die Kategorien systematisch ausgearbeitet wurden (vgl. Muckel 2011, S. 345), indem Verbindungen zwischen der Kategorie und den Codes ermittelt wurden (vgl. Strauss/Corbin 1996, S. 76). Die Methodik der Grounded Theory findet als Werkzeugkasten häufig Anwendung in der Rekonstruktion von Deutungsmustern (vgl. u. a. Keller 2008, Keller 2011b, Sitter 2016, Brandmayr 2018), da sie mit ihrem Codierverständnis eine allgemeinere Reflexion und übertragbare Systematisierung der Zwischenschritte anbietet, durch die eine diskursbezogene Rekonstruktion von Deutungsmustern ermöglicht wird (vgl. Keller 2008, S. 95). Da Deutungsmuster nicht sichtbar sind und deshalb rekonstruiert werden müssen (vgl. Ullrich 2020, S. 16) und dieser Prozess eine Offenheit bezüglich der Herangehensweise an den Untersuchungsgegenstand verlangt, erscheint die Methodik der Grounded Theory besonders geeignet, da es zum einen darum geht, Ursachen und Bedingungen eines Phänomens zu untersuchen (vgl. Bormann 2011, S. 167) und dies, zum anderen, mit Blick auf „die Subjektseite der Erkenntnis in alltagsweltlichen [...] Zusammenhängen“" (Breuer/Muckel/Dieris 2019, S. 38) geschieht.

Grundsätzlich wäre es auch möglich, hier mit der dokumentarischen Methode (vgl. Bohnsack 2003) zu operieren (vgl. Keller 2011b, S. 80), da diese im deutschen Sprachraum, neben der Grounded Theory, häufig zur Rekonstruktion von Deutungsmustern angewandt wird (vgl. Ullrich 2020, S. 21) und deshalb auch methodisch eine Anschlussfähigkeit zu anderen Analysen gegeben wäre. Wie aber bereits dargelegt wurde, orientiert sich diese Studie an Berger und Luckmanns wissenssoziologischem Verständnis („Allerweltwissen“) und nicht an der Wissenssoziologie Mannheims („elaborierte Wissensformen“), die aber eine wesentliche metatheoretische Grundlage der dokumentarischen Methode darstellt (vgl. Asbrand 2011, S. 2). Um also eine überkomplexe Kombination von Theoriesträngen zu vermeiden, wird in der Folge auf die Anwendung der dokumentarischen Methode zugunsten der Methodik der Grounded Theory für die Deutungsmusteranalyse verzichtet.

Die Interviews wurden im April und Mai 2021 per Skype und Zoom geführt und mithilfe des Programms „f4“ transkribiert. Um der im ersten Abschnitt erwähnten regionalen Perspektive gerecht zu werden, war es für die Auswahl der Schulen wichtig, dass sie sich beide im Großraum Stuttgart (genauer: in zwei Kleinstädten nahe Stuttgart) befinden. Zudem wird in beiden Schulen ausschließlich die Sekundarstufe I angeboten; es werden also die Klassen 5 bis 10 beschult. Um die Aussagen der Lehrkräfte noch vergleichbarer zu gestalten, wurde darauf geachtet, dass beide Schulen das gleiche Lern-Management-System verwenden - in diesem Fall Microsoft Teams ${ }^{10}$. Den zehn Lehrer*innen wurde umfassende Anonymität zugesichert, weshalb die Lehrkräfte jeweils durch einen Buchstaben A - J ${ }^{11}$ pseudonymisiert werden. Gesagt

\footnotetext{
${ }^{9}$ Bohnsack verwendet den Begriff „Orientierungsmuster“ anstelle des „Deutungsmusters“, wobei inhaltlich aber deutliche Parallelen feststellbar sind (vgl. Ullrich 2020, S. 20).

${ }^{10}$ An den Schulen, an denen die befragten Lehrkräfte arbeiten, wird Microsoft Teams nicht nur als Videokonferenztool, sondern auch als Lern-Management-System verwendet. Beispielsweise können auf Microsoft Teams, wie auch auf Moodle, von den Lehrkräften Aufgaben für die Schüler*innen eingestellt werden. Nach einer erfolgten Bearbeitung können die Lehrkräfte den Schüler*innen ein Feedback geben.

${ }^{11}$ Es werden nicht alle Befragten zitiert, das würde den Rahmen des Beitrages sprengen. Zu Gunsten einer genderneutralen Analyse wird auf die Nennung des Geschlechts der befragten Lehrkräfte verzichtet. Zugleich geben jüngere
} 
werden kann hier aber, dass alle interviewten Lehrkräfte mindestens eine Klassenleitung haben. Aufgrund dessen ist davon auszugehen, dass eine tiefergehende Bindung zu deren Schüler*innen besteht, als das bei reinen Fachlehrkräften der Fall wäre und sich auf das Ergebnis der Studie auswirken könnte. Die mediale Ausstattung ihrer Schule empfinden die Teilnehmer*innen, relativ zu anderen Schulen gut, im Verhältnis zum technisch-möglichen Standard allerdings unzureichend. Ein konsequent umgesetztes digitales pädagogisches Bildungskonzept haben beide Schulen nach Aussagen der Befragten nicht.

\section{Analyse der Deutungsmuster der Lehrer*innen}

Die im Folgenden angeführten Zitate sind immer exemplarisch zu verstehen. Der zitierte Fall erschien während der queranalytischen Kontrastierung als besonders geeignet, um das jeweilige rekonstruierte soziale Deutungsmuster der Lehrer*innen zu veranschaulichen.

\section{Deutungsmuster bezüglich medialer Ausstattung und der aufgetretenen Herausforderungen}

Diesen Teil einleitend, steht eine Explikation einer Lehrkraft, bezüglich auftretender unterrichtlicher Herausforderungen während des Notfall-Fernunterrichts: „/ähm/, ja (1) bei den Schülern war natürlich komplett die Ausstattung, lähm/, das Problem, was ja irgendwie auch zu erwarten war, dass viele auch kein Endgerät haben. (1) Oder, im Prinzip keine entsprechende Internetverbindung. Das ja irgendwann ausgeglichen worden ist, mit den i-Pads (...) Viele arbeiten ja auch mim Handy (1) nach wie vor, obwohl wir des ja alles anbieten (2) und des kann ja gar ned funktionieren "(Lebrer*in A, 36) ${ }^{12}$. Zunächst wird deutlich, dass die mediale Ausstattung der Schüler*innen mit Endgeräten und Internetverbindungen, für die Lehrkräfte, einen zentralen Stellenwert für einen gelingenden - „funktionierenden“ - Unterricht einnimmt. Der Fernunterricht war für die Lehrkräfte demnach vor allem aufgrund der nicht vorhandenen Ausstattung unzureichend. Durch den Verweis auf „das Problem“, das daraus besteht, dass eben keine Endgeräte oder ausreichende Internetverbindung vorhanden war, kann auf eine auftretende unterrichtsmethodische Herausforderung geschlossen werden: digital interaktiv gestaltete Arbeitsblätter können, beispielsweise auf einem Handy, von den Schüler*innen genauso wenig gut bearbeitet werden, wie ein analoges Arbeitsblatt, da ein solches ohne vorhandenen Drucker schlichtweg nicht ausgedruckt werden kann. Die Handlungsmöglichkeiten der Lehrkräfte erleben sie deshalb als sehr eingeschränkt. Professionstheoretisch kann hier auf den Gedanken einer effizienten Zeitnutzung (vgl. Helmke 2012) ${ }^{13}$ geschlossen werden. In

\footnotetext{
Lehrkräfte, im Gegensatz zu älteren Lehrkräften, häufiger an, sich gut mit Lern-Management-Systemen auszukennen (vgl. Drossel et al. 2019, S. 227). Da der Fernunterricht online auf eben solchen stattfindet, wird beim ersten Zitat einer Lehrkraft das Alter mitangegeben. Beispiel: (Lehrer*in A, 35).

${ }^{12}$ Für die Transkription wurde eine einfache literarische Umschrift verwendet, da dies für die Rekonstruktion sozialer Deutungsmuster ausreicht (vgl. Ullrich 2020, S. 122). Dabei wurden Betonungen fett notiert, die Zeiten der Satzunterbrechungen in Klammern angegeben und etwaige „äh“ o.ä. durch Schrägstriche gerahmt.

${ }^{13}$ Explizit auf Helmke wird hier im Zuge der Interpretation rekurriert, da dessen Überlegungen einen zentralen Stellenwert in der zweiten Phase der Lehrer*innenbildung in Baden-Württemberg einnehmen (vgl. hierzu exemplarisch die Literaturliste des Fachbereichs Pädagogik des Seminars für Ausbildung und Fortbildung der Lehrkräfte Schwäbisch Gmünd in SAFL 2016). Es wird deshalb davon ausgegangen, dass die interviewten Lehrkräfte - bewusst oder unbewusst - in ihren Begründungen darauf Bezug nehmen.
} 
diesem Paradigma „funktioniert“ Unterricht (unter anderem) dann gut, wenn dieser nicht durch eine unvorbereitete Lernumgebung gestört wird - und darauf haben Lehrer*innen in dieser Unterrichtsform nur sehr wenig Einfluss. Die Explikation der Lehrkraft beinhaltet außerdem den normativ aufgeladenen Begriff „natürlich“. Auffallend dabei ist die Verwendung des Kommentaradverbs „natürlich“, vor allem deshalb, da dadurch die entstandenen negativen Konnotationen bezüglich des Fernunterrichts für die Lehrkraft scheinbar unausweichlich waren. Zum anderen lässt sich der Begriff „natürlich“, wie auch „komplett“ und „erwarten“ mit Link (vgl. 2006) als für eine digitale Gesellschaft, untypisch anmutenden Normalismus lesen: Auf einer diskursiven Ebene lässt sich daraus schließen, dass diese Wahrnehmung von Normalität (Schüler*innen haben keine mediale Ausstattung) „durch [Diskurse] produziert und reproduziert werden" (Link 2006, S. 60).

Die nachfolgende Explikation einer Lehrkraft zu sozio-ökonomischen Herausforderungen im Notfall-Fernunterricht, stellt die wahrgenommene Situation prägnant dar: „[K]ein eigenes Zimmer, insgesamt prekäre familiäre Verbältnisse. $/ A ̈ b m /(1)$ Kind is allein zubause, als Beispiel oder vier Söbne, alle unter 18 sind allein zubaus' im Onlineunterricht. Oder (1) äbnliches" (Lebrer* in D, 56). Diese Aufzählung, in der die mediale Ausstattung nicht erwähnt wird, lässt sich in die Richtung interpretieren, dass in der Deutung der Lehrkräfte, Kinder aus sozio-ökonomisch schwächeren Familien, nicht nur bedingt durch die fehlende mediale Ausstattung, sondern auch durch ein nicht lernförderliches Klima (vgl. Helmke 2012) im Notfall-Fernunterricht als benachteiligt angesehen werden. Auch die Nennung der „prekären familiären Verhältnisse" in Verbindung mit dem Kind, das allein zuhause ist, verdeutlicht eine gedeutete Benachteiligung. Zum einen ist es nicht lernförderlich, wenn mehrere Personen in einem Raum - und dazu noch bei unzureichender medialer Ausstattung - an unterschiedlichen Unterrichtsstunden teilnehmen. Zum anderen ist es jedoch auch nicht lernförderlich, wenn ein Kind in der Zeit des Unterrichts andauernd „allein zuhause“ ist, da hier das psychologische Grundbedürfnis nach „sozialer Eingebundenheit“(Deci/Ryan 1993, S. 229) nicht befriedigt werden kann, was aber allgemein für die Motivation von Menschen und damit auch für die Lernmotivation - und in der Folge für den Lernerfolg - von Schüler*innen eine wichtige Rolle spielt.

Außerdem lässt sich noch eine von den Lehrkräften wahrgenommene Herausforderung, bezogen auf den sozialen Aspekt des Unterrichts, rekonstruieren. Die folgenden zwei Begründungen der zitierten Lehrkräfte wurden auf die Nachfrage, warum sie den Notfall-Fernunterricht als unzureichend empfinden, gegeben: „Das, was Unterricht ausmacht, is ja so gar nicht möglich, also (1) das ganze Zwischenmenschliche und so. "(Lehrer*in I, 33). und „(...) dass du überhaupt kein Feedback kriegst. Also Feedback nich'im Sinne von ,Hey Sie mach'n des so toll, sondern du kriegst ja null Reaktion, null, weil die Kameras und Mikros in aller Regel aus sind, was ja nicht anders gebt, wenn 30 Leute /äb/ konferieren "(Lebrer*in D). An dieser Stelle wird die Uneinigkeit der Lehrkräfte (unabhängig von ihrem Alter) mit dieser Form des Unterrichts deutlich. Dem Fernunterricht wird, mit dem Verweis darauf, dass Interaktion und Zwischenmenschlichkeit dort nicht gegeben sind, diese aber den Unterricht für die Lehrkräfte ausmachen, sogar abgesprochen „Unterricht“ zu sein. Daraus lässt sich ableiten, dass die befragten Lehrer*innen in ihrem Unterricht zuerst eine erzieherische oder gar persönlichkeitsbildende Dimension sehen und dem nachgereiht eine Qualifikationsfunktion (vgl. Fend 2008), da die 
Dimension der Stoffvermittlung in diesem Urteil der Lehrkräfte keine Betrachtung findet. Es tritt so prägnant die Frage nach der technischen Reproduzierbarkeit des sozialen Raums (vgl. Barberi/Swertz 2020, S. 80) auf. In der Deutung der Lehrkräfte findet zwischen ihnen und ihren Schüler*innen keine so intensive soziale Interaktion statt, wie das in analoger Form des Unterrichts für sie wohl möglich wäre. Besonders ausgeprägt nachzuvollziehen, ist diese Deutung in der Betonung „,null Reaktion, null“. Zurückzuführen ist das wiederum auf den eigentlichen Alltag der Lehrer*innen, in dem sie während des Unterrichts beispielsweise die Körpersprache der Schüler*innen deuten können und diese Möglichkeit des „crowd-readings ${ }^{\text {“14 im }}$ Online-Unterricht für sie nicht stattfinden kann.

Diese (Re-)konstruktionen können hier zu einer Deutung verdichtet werden: Lehrer*innen deuten die großen Herausforderungen im Notfall-Fernunterricht zum einen in der mangelnden medialen Ausstattung und den familiären Verhältnissen der Schüler*innen sowie zum anderen in der fehlenden Interaktion und Zwischenmenschlichkeit. Wobei den Lehrkräften zufolge besonders die Interaktion und die Zwischenmenschlichkeit - in Form des gemeinsamen in-der-Schule-Seins von Lehrkraft und Schüler*innen - als die den Unterricht konstituierenden pädagogischen Momente aufgefasst werden.

\section{Deutungsmuster bezüglich der Potentiale digitaler Lernangebote}

Im Folgenden findet sich eine Begründung einer Lehrkraft wieder, in der darauf eingegangen wird, warum im Fernunterricht „doch nicht alles schlecht war":

„Ich denk, ich denk die Digitalisierung, diese Medien, mit denen wachsen die grade auf. (1) In all'n Bereichen der Berufswelt wird die, taucht die Digitalisierung auf und wird denen begegnen. Und da isses ja schon ein Geschenk, dass wir damit jetzt konfrontiert wurden. Und da kann man, des kann man (1) ja beibebalten. Ich glaub, des bat eine große Bedeutung, der Umgang mit diesen Medien, /öh/, dass man nicht nur damit zocken kann und Netflix kucken. Die baben bei mir zu dritt an einer PowerPoint gearbeitet. Des war für die auch voll der Aba-Effekt /öh/, also, (1) sensationell, wie dann halt im Berufsleben auch, Erklärvideos habe ich auch machen lassen "(Lebrer* in C, 53). In dieser Aussage wird die notwendig stattfindende und intensivere Beschäftigung mit dem produktiven Umgang (hier mit dem Beispiel „an PowerPoint gearbeitet" oder „Erklärvideos") mit digitalen Medien im Unterricht hervorgehoben. Gleichzeitig wird der alltägliche sowie der spätere ökonomische Nutzen, der durch diesen produktiven Umgang für die Schüler*innen entsteht, dargestellt. Die Betonung des produktiven Umgangs wird durch das Hervorheben der Tatsache, dass „man nicht nur damit zocken kann und Netflix kucken “, sehr deutlich. Mit dieser Deutung subjektivieren ${ }^{15}$ Lehrkräfte Schüler*innen dahingehend, dass den Schüler*innen eine produktive Beschäftigung mit digitalen Medien „von sich aus“ zunächst nicht zugetraut wird. Die Schüler*innen sind damit im Verständnis

\footnotetext{
${ }^{14}$ Dieser Begriff ist der Sprache von Bühnenkünstler*innen entlehnt, die während ihres Auftritts die Stimmung des Publikums lesen und daraufhin ihr Programm anpassen können, also mit dem Publikum nonverbal interagieren, bzw. auf das Publikum eingehen.

${ }^{15}$ Unter Subjektivierung wird hier, im Anschluss an Butler, die Beeinflussung der Selbstidentität eines Subjekts (vgl. Butler 2001, S. 8 f.) - hier: die der Schüler*innen - verstanden, was Lehrkräfte durch ihre Adressierungspraktiken (vgl. Balzer \& Ricken 2010) und durch ihre Stellung im Konstrukt Schule andauernd (bewusst oder unbewusst) tun.
} 
der Lehrer*innen im Wesentlichen Konsument*innen von digitalen Medien. Gleichzeitig wird den Schüler*innen aber auch zugestanden, lernfähig zu sein - deutlich am „Aha-Effekt“. In der Folge deutet das „Geschenk“ auf eine weitere Subjektivierung hin: die Schüler*innen werden von den Lehrer*innen in einer möglichen späteren Rolle auf dem Arbeitsmarkt verortet. In der Folge ist ein weiteres subjektivierendes Exponat die Bezugnahme auf „die Berufswelt“, die zwar nicht weiter spezifiziert wird, in der Deutung aber eine zentrale Rolle spielt, da hier die Begründung für die „Potentiale“ (wofür?) verortet werden kann. Interessant ist das besonders deshalb, da der Fluchtpunkt des Arguments der befragten Lehrkräfte augenscheinlich in der Vorbereitung auf die Berufswelt liegt und nicht auf den aktivierenden Potentialen des produktiven Umgangs mit Medien für das Lernen selbst. Dies deutet darauf hin, dass die Möglichkeit, produktiv und digital im Unterricht zu arbeiten, nicht gegeben war, andernfalls wäre der erwähnte „Aha-Effekt“ nicht erst in der Zeit des digitalen Notfall-Fernunterrichts aufgetreten. In der Deutung der Lehrkräfte wurden demnach diese möglichen Potentiale bisher verschenkt (vgl. hierzu auch Knaus 2018, S. 12 ff.).

Verdichtend lässt sich sagen, dass Lehrer*innen Schüler*innen hinsichtlich der Nutzung digitaler Medien als „lernfähige Konsument*innen“ betrachten, die für „die Berufswelt“ fit gemacht werden müssen.

\section{Deutungsmuster bezüglich der Verantwortungszuschreibung}

Bezüglich der Verantwortungszuschreibung in Fragen der medialen Ausstattung lässt sich ein enger Zusammenhang zwischen den Lehrkräften und der den Lehrkräften vorgesetzten Instanzen nachweisen, die von den Lehrkräften kritisch hinterfragt wird. Deutlich wird das an den folgenden Interviewauszügen: Eine Lehrkraft gibt auf die Frage, wem die Verantwortung für einen guten Fernunterricht zugeschrieben wird, folgende Antwort: „Federführend isch natürlich schon des Land für die Ausstattung verantwortlich und jeder Lebrer muss dann da fortgebildet sein, wie das funktioniert. (...) es weiß halt keiner so richtig, wie's gebt. (...) und gute Fortbildungen dazu gibt's ja grad' net." (Lebrer*in B). Die Verantwortlichkeiten werden hier imperativ als Norm eingeführt: Das Land (also das Kultusministerium) „,ist“ für die digitale Ausstattung und für die Fortbildungen in diesen Bereichen verantwortlich, die jeder Lehrkraft zugutekommen muss. Der Notfall-Fernunterricht verlief in der Deutung der Lehrer*innen deshalb nicht lernzielführend, da das Land der zugeschriebenen Verantwortung nicht ausreichend nachkam, da die Angebote von den Lehrer*innen - wieder normativ - als „nicht gut“ angesehen wurden. Die Lehrer*innen verstehen ihr Handeln als Produkt der Situation: Würde es gute Fortbildungen geben, dann wäre der Notfall-Fernunterricht zumindest in didaktischmethodischer Hinsicht lernzielführender.

Im Kontrast zu dieser Deutung steht das folgende Deutungsmuster: „Dass wir uns was beim Tun überlegen ist ja nicht gewollt. Man [muss] erst ein Konzept, ein dreißigseitiges, erstellen und erst wenn des irgendwelchen Ansprüchen genüge tut, dann bekommt ibr's Geld. (...) Kein Vertrauen in die Schulen beziehungsweise die handelnden Personen, sondern erstmal Konzept, Kontrolle des Konzepts und dann kriegste's Geld" (Lebrer*in D). Auch in dieser Explikation kann eine deutliche normative Orientierung festgestellt werden. Allerdings wird hier die vorgesetzte Instanz als aktive und intransparente Verhinderung eines guten Fernunterrichts 
gedeutet. Der im Interviewausschnitt beschriebene institutionell-hierarchische Normalismus (erst ein Konzept zu schreiben, das dann - für die Lehrkräfte - intransparent kontrolliert wird) wirkt auf die Lehrer*innen deshalb befremdlich, da ihnen Entscheidungskompetenz, bezogen auf die Nöte ihres Unterrichts, nicht zugetraut wird - und das, obwohl ihnen das Unterrichten selbst als „handelnde Personen“ offensichtlich zugetraut wird.

Für beide Explikationen lässt sich allerdings konstatieren, dass die Lehrer*innen sich selbst nicht aus der Verantwortung nehmen: Für den ersten Interviewausschnitt lässt sich eine Hilfslosigkeit, gepaart mit einem Willen zur Gestaltung eines guten Fernunterrichtes erkennen und für den zweiten ein selbstbewusstes Vertrauen in die eigenen beruflichen Fertigkeiten, die aber vom Arbeitgeber verhindert werden.

Die hier rekonstruierten Deutungsmuster zeigen eine pragmatische Kausalkette in der Verantwortungszuschreibung: Wenn das Kultusministerium - und alle anderen, den Schulen vorgesetzten Instanzen - ihren Verantwortlichkeiten nicht nachkommen, kann ein guter Unterricht nur schwer realisiert werden.

\section{Deutungsmuster bezüglich des Stellenwertes der Digitalisierung für die Bildung}

Der Einsatz digitaler Medien im Unterricht wird von den Lehrer*innen grundlegend befürwortet. Zum einen mit dem Nutzen-Argument, dass aufgrund des Umstandes, dass ein Verständnis für digitale Medien „in der Berufswelt immer wichtiger is, also müssen die Schüler da schon auch gut drauf vorbereitet werden" (Lebrer*in B). Zum anderen mit dem Argument der Persönlichkeitsentwicklung, aufgrund der Tatsache, dass „die digitalen Medien n wabnsinnigen Stellenwert im Leben von, von Heranwachsenden baben "(Lebrer*in D), weshalb es in der Deutung der Lehrer*innen besonders wichtig sei, dass Schüler*innen einen „kritischen Umgang beigebracht kriegen, /äb/ (2) oder zumindest des ab und an au hinterfragen " (Lehrer $^{*}$ in E, 46) und im Unterricht thematisiert werde, "wie ich mit digitalen Medien umgeh und auch in ibrem Sozialem, also äbm ich find auch grad die Gefabren die die Digitalisierung bietet, da muss man Schüler schon auch drauf hinweisen und da muss man sie drauf vorbereiten" (Lebrer*in B). Anhand dieser Interviewausschnitte können zwei normative Deutungslinien die Schüler*innen müssen auf etwas vorbereitet werden - herausgearbeitet werden. Zum einen eine ökonomisch konnotierte Deutung, die den Einsatz von digitalen Medien im Unterricht damit begründet, dass das für die Schüler*innen wichtig sei, um sich später auf dem Arbeitsmarkt zurechtzufinden. Zum anderen eine persönlichkeitsbildende Deutung, die sich gleichzeitig medienkritisch verstehen lässt, da hier „die Gefahren“, die durch digitale Medien an die Schüler*innen herangetragen werden - und für die die Schüler*innen durch den Unterricht, zu sensibilisieren sind - in den Vordergrund treten. Weiter sehen sich Lehrer*innen „nicht als digitale Skeptiker" (Lebrer*in F, 30). Der Schwerpunkt in deren Deutung ihrer Profession liegt lediglich woanders: Den Lehrer*innen gehe es "grundsätzlich [...] ja au um die Erziebung zu mündigen Bürgern" (Lebrer*in D) und darum, dass Schüler*innen „mit einer eigenen Persönlichkeit aus der Schule entlassen werden "(Lebrer*in A). Im Bildungsprozess der Schüler*innen deuten Lehrer*innen digitale Medien lediglich als „Beiwerk“ (Lebrer*in F). Des Weiteren deuten die Lehrkräfte diesbezüglich ein großes Problem in digitalen Bildungsprozessen, wie sie im Fernunterricht stattgefunden haben, da dort für die Lehrkräfte - wie schon 
vorhin - digital „dieses Zwischenmenschliche fehlt"(Lebrer*in B). Diese medienkritische Deutung wird nochmals besonders offenbar, wenn Lehrkräfte den öffentlichen Diskurs zu „digitale Medien und Schule“ verarbeiten: „Der Trugschluss [entsteht CS], je digitaler wir sind, desto besser ischs, und dann wird lähm/ eine Vorzeigeschule in den Medien zeigt, die eine super digitale Ausstattung haben /ähm/und des dann plötzlich die (1) beste Schule Deutschlands wird. Das mag sein, dass das eine super Schule isch und die einen super Job macht, aber nicht nur allein deswegen, weil sie digital super ausgestattet isch" (Lebrer*in A). Wenngleich nicht weiter spezifiziert wird, wann eine Schule einen „super Job“ macht, so macht sie das in der Deutung der Lehrkräfte nicht allein durch eine gute Ausstattung. Aus der Perspektive der Lehrkräfte kann der Einsatz von digitalen Medien im Unterricht kein Selbstzweck sein. Digitale Medien werden lediglich als ein mögliches Mittel für einen guten Unterricht angesehen ${ }^{16}$.

Schließlich lässt sich hier zusammenfassen, dass Lehrer*innen den Stellenwert des digitalen Wandels für die Bildung in zwei Dimensionen deuten. Erstens in der ökonomisch konnotierten Deutung, in der digitale Medien einen späteren Nutzen für die Schüler*innen auf dem Arbeitsmarkt haben und zweitens in einer persönlichkeitsbildenden Konnotation, wobei der kritische Umgang mit denen durch digitale Medien vermittelten Inhalte leitend ist.

\section{Diskussion}

Mit Blick auf die rekonstruierten Deutungsmuster der Lehrer*innen stellt sich die „Frage nach der technischen Reproduzierbarkeit des sozialen [...] Raums"(Barberi/Swertz 2020, S. 80). Im Zuge des Notfall-Fernunterrichts sehen die Lehrer*innen die Möglichkeitsbedingungen für erfolgreiches pädagogisches Handeln im technisch erzeugten sozialen Raum nur sehr eingeschränkt gegeben - nämlich nur für den Fall der Stoffvermittlung. Damit ist wohl einem rein curricularen Verständnis von Bildung Genüge getan. Allerdings können wesentliche Merkmale eines "guten Unterrichts“ (vgl. u. a. Helmke 2012; Meyer 2016, beide zentral in der Lehrer*innenausbildung in BW, vgl. SAFL 2016) nicht gewährleistet werden, da z. B. auf Determinanten wie ein lernförderliches Klima, den Anteil echter Lernzeit oder die vorbereitete Lernumgebung (vgl. Meyer 2016) nur sehr eingeschränkt Einfluss genommen werden kann. Erschwerend kommt hier wohl die erwähnte, technisch-induzierte „Null-Reaktion“ (Deutungsmuster zur „Herausforderung“) hinzu, wodurch es zum Fehlen der, in der Deutung der Lehrkräfte, für den Unterricht so wichtigen Interaktion und Zwischenmenschlichkeit kommt. Dieser Logik folgend entstehen Effekte, die sich aus der Sicht der Lehrkräfte u. U. nicht förderlich auf die Entwicklung der Schüler*innen auswirken können. So fehlt im Notfall-Fernunterricht beispielsweise das für die Motivation wichtige psychologische Grundbedürfnis der sozialen Eingebundenheit (vgl. Deci/Ryan 1993, S. 229) in einen Klassenverband - was besonders bei Schüler*innen in der SEK-1 eine nicht unbedeutende Rolle spielt (vgl. Bakadorova/Raufelder 2018). Ob in dieser Form des Fernunterrichts nach Deci und Ryan (1993)

\footnotetext{
${ }^{16}$ Anzumerken ist an dieser Stelle, dass sich diese Deutung allgemein auf den zugeschriebenen Bildungswert von digitalen Medien bezieht und nicht auf den Fernunterricht, der ja - wie bereits mehrfach erwähnt - ohne digitale Medien überhaupt nicht möglich wäre.
} 
von einem realisierten Erleben von Autonomie und Kompetenz gesprochen werden kann, ist fraglich. Es ist wohl zuvörderst davon auszugehen, dass der Fernunterricht als Form des Unterrichts eher nicht fortgeführt wird und als notwendiges Übel in Erinnerung bleibt. Aufgrund der jetzigen Erfahrungen - und der Tatsache, dass Szenarien, in denen Interaktion nur medial stattfindet, in der Zukunft der Schüler*innen nicht unwahrscheinlicher werden, sind jedoch medienpädagogische - oder genauer: mediendidaktische - Überlegungen angebracht. Zielführend erscheint dabei die Auseinandersetzung mit den Möglichkeitsbedingungen der technischen Reproduzierbarkeit des Sozialen im Unterricht. Das Ergebnis könnte dabei einerseits ein realistischer Gehalt dessen sein, was digitale Medien für die soziale Dimension des (Fern-)Unterricht leisten könn(t)en und andererseits gerade nicht in der Lage sind zu leisten. Eine Frage, deren Bearbeitung sich im Zuge dessen vermutlich lohnt, ist die nach den konstitutiven Formen der digitalen Interaktion und Zwischenmenschlichkeit - auch hier lässt sich wieder die Selbstbestimmungstheorie von Deci und Ryan bemühen. Hier scheint es, als ob genau dazu mediendidaktische Überlegungen nötig sind: Wie können Lehrende und Lernende zu dieser Form der Kommunikation motiviert werden? Ganz im Sinne der unterrichtlichen Praxis sei hier angemerkt, dass Fragen, die den „Umgang mit Heterogenität“ (vgl. Bohl/Budde/Rieger-Ladich 2017) betreffen, noch nicht umfassend beleuchtet wurden.

Des Weiteren ist ein hohes Maß an auf die Handlungsprobleme des Alltags bezogenem Pragmatismus auszumachen. Demnach lässt sich das Bild zeichnen, dass Medien - ob digital oder analog - von den Lehrer*innen dann gerne genutzt werden, wenn sie funktionieren - was wohl auch eine Erklärung für die stellenweise unveränderte Beliebtheit des Schulbuches und der Kreidetafel ist, die eben ganz elementar: funktionieren. Wenn also Schulen einen digitalen Wandlungsprozess durchlaufen sollen, dann ist das - gemäß dem Ergebnis der vorliegenden Studie - zum einen vor allem dann zu erreichen, wenn gewährleistet ist, dass Lehrer*innen damit auch lernzielorientiert unterrichten können und den Schulen zum anderen ermöglicht wird, funktionierende digitale Medien in den Unterricht zu integrieren. Das Deutungsmuster, dass digitale Medien lediglich als Beiwerk für den Unterricht betrachtet werden, erscheint dabei auf den ersten Blick (vermutlich) als Barriere. Allerdings darf hier nicht außer Acht gelassen werden, dass die Lehrerkräfte den Einsatz digitaler Medien im Unterricht auch als Chance deuten, Medienkompetenz sowie ein Verständnis für Medienkritik aufzubauen, was den Schüler*innen persönlich sowie beruflich zugutekommt.

Für die allgemeine schulpädagogische Didaktik und Methodik geht aus den rekonstruierten Deutungsmustern der stellenweise anmutende Antagonismus aus Fortschritt und Innovation hervor. Ein „interaktives Arbeitsblatt“ ist zwar durchaus innovativ, aber lediglich ein digitales Abbild eines analogen Mediums - um hier nochmal McLuhan zu bemühen: die Botschaft ist die gleiche und damit nicht fortschrittlich, da die Frage, worin der Mehrwert für das Lernen besteht, damit noch nicht beantwortet ist ${ }^{17}$. Vor allem dann nicht, wenn bekannt ist, dass sich der auftretende motivationale Effekt des Einsatzes von für die Schüler*innen „neuer Medien“ anfangs auf die neue Technik selbst bezieht, dieser Neuigkeitseffekt sich aber relativ schnell abnützt (vgl. Kerres 2003, S. 3).

\footnotetext{
${ }^{17}$ Vielversprechenderes (beispielsweise adaptive Lernsysteme) findet sich bei Macglichrist (vgl. 2018).
} 
Methodologisch-methodisch bleibt an dieser Stelle zu erwähnen, dass die hier rekonstruierten Deutungsmuster keine universale Gültigkeit beanspruchen können. Daran anschließend wäre deshalb interessant, wie Lehrkräfte an Grundschulen oder an Gymnasien die entstandenen Disruptionen wahrgenommen haben. Für andere Bundesländer gilt gleiches - dort die Sekundarstufen-I-Schulen miteingeschlossen. Aus der Perspektive der regionalen Schulentwicklung könnte die Vorgehensweise sicherlich noch verfeinert werden: Welche Deutungsmuster sind bei Lehrkräften an Stadt und an Landschulen vorhanden? Und/Oder: Welche Implikationen können daraus für die Lehrer*innenbildung gezogen werden? Die Möglichkeiten an Analyse- und Schlussfolgerungsoptionen sind mit diesem Beitrag also sicherlich nicht erschöpft.

Inhaltlich abschließend kann hier festgehalten werden, dass digitale Lernmedien für den (analogen) Unterricht eine untergeordnete Rolle im Professionsverständnis der teilnehmenden Lehrer*innen spielen. Den Wert von digitalen Medien für eine gelungene Bildung von Schüler*innen sehen die Lehrer*innen zum einen darin, dass Schüler*innen durch das Arbeiten mit digitalen Medien tatsächlich medienkompetent werden, wobei die Schüler*innen ganz im Sinne eines kant'schen „sapere aude“ zum kritischen Denken bewegt werden sollen. Zum anderen in einer ökonomischen Verwertbarkeit dieses Wissens. Allerdings sehen die Lehrer*innen den Wert des Unterrichts vor allem in der Persönlichkeitsbildung der Schüler*innen und dabei sind digitale Medien aller Art für die Lehrer*innen lediglich „Beiwerk“ (4.). Woraus als Implikation für die Aus- und Fortbildung der SEK-I-Lehrkräfte in Baden-Württemberg geschlossen werden kann, dass es die Aufgabe der Medienpädagogik und Mediendidaktik ist, den Wert des Einsatzes digitaler Medien hinsichtlich der Persönlichkeitsbildung zu vermitteln. Das Konzept der Aktiven Medienarbeit bietet dabei einige Ansatzpunkte.

\section{Literatur}

Arnold, Rolf(1983): Deutungsmuster. Zu den Bedeutungselementen sowie den theoretischen und methodologischen Bezügen eines Begriffs. In: Zeitschrift für Pädagogik, 6, S. 893-912.

Asbrand, Barabra (2011): Dokumentarische Methode. URL: fallarchiv.uni-kassel.de/backup/wpcontent/plugins.old/lbg_chameleon_videoplayer/lbg_vp2/videos//asbrand_dokmethode_ofas.pdf (25.07.2021).

Bakadorova, Olga/Raufelder, Diana (2018): The essential role of the teacher-student relationship in students' need statisfaction during adolescence. In: Journal of Applied Develeopmental Psychology, 58, pp. 57-65.

Balzer, Nicole/Ricken, Norbert (2018): Anerkennung als pädagogisches Problem. Markierungen im erziehungswissenschaftlichen Diskurs. In: Schäfer, Alfred/ Thompson, Christiane (Hrsg.): Anerkennung. Paderborn: Ferdinand Schöningh, S. 35-87.

Barberi, Alessandro/Swertz, Christian (2020): Doing Digital - Ein Beitrag zur Medienkompetenzvermittlung für Entscheidungsimpulse setzende Akteur*innen. In: Dander, Valentin/Bettinger, Patrick/Ferraro, Estella/Leineweber, Christian/Rummler, Klaus (Hrsg.): Digitalisierung - Subjekt Bildung. Kritische Betrachtungen der digitalen Transformation. Berlin/Toronto: Opladen, S. 77-96. 
Berger, Peter/Luckmann, Thomas (2013): Die gesellschaftliche Konstruktion der Wirklichkeit. Eine Theorie der Wissenssoziologie. Berlin: Fischer.

Brandmayr, Michael (2018): Dispositive des Lernens. Analyse der Formierung schulischer Lernprozesse unter ideologiekritischen Aspekten. Wiesbaden: Springer VS.

Bremm, Nina/Racherbäumer, Kathrin (2020): Dimensionen der (Re-)Produktion von Bildungsbenachteiligung in sozialräumlich deprivierten Schulen im Kontext der Corona-Pandemie. In: Fickermann, Detlef/Edelstein, Benjamin (Hrsg.): „Langsam vermisse ich die Schule...“ - Schule während und nach der Corona-Pandemie. Die Deutsch Schule - Zeitschrift für Erziehungswissenschaft, Bildungspolitik und pädagogische Praxis, Beiheft, 16, Münster/New York: Waxmann, S. 202-215.

Breuer, Franz/Muckel, Petra/Dieris, Barbara (2019): Lehrbuch: Reflexive Grounded Theory. Eine Einführung für die Forschungspraxis. Wiesbaden: Springer VS.

Bohl, Thorsten/Budde, Jürgen/Rieger-Ladich, Markus (2017): Umgang mit Heterogenität in Schule und Unterricht. Grundlagentheoretische Beiträge, empirische Befunde und didaktische Reflexionen. Bad Heilbrunn: Klinkhardt.

Bohnsack, Ralf (2003): Rekonstruktive Sozialforschung. Einführung in qualitative Methoden. Opladen/Toronto: Verlag Barbara Budrich.

Bormann, Inka (2011): Zwischenräume der Veränderung: Innovation und ihr Transfer im Feld von Bildung und Erziehung. Wiesbaden: Springer VS.

Butler, Judith (2001): Psyche der Macht. Das Subjekt der Unterwerfung. Frankfurt am Main: Suhrkamp.

Deci, Edward L./Ryan, Richard M. (1993): Die Selbstbestimmungstheorie der Motivation und ihre Bedeutung für die Pädagogik. In: Zeitschrift für Pädagogik, 39 - 2, S. 223-238.

Drerup, Johannes/Schweiger, Gottfried (2020): Bildung und Erziehung im Ausnahmezustand. Philosophische Reflexionsangebote zur COVID-19 Pandemie. Darmstadt: wbg Academic.

Drossel, Kerstin/Eickelmann, Birgit/Schaumburg, Heike/Labusch, Amelie (2019): Nutzung digitaler Medien und Prädiktoren aus der Perspektive der Lehrerinnen und Lehrer im internationalen Vergleich. In: Eickelmann, Birgit/Bos, Wilfried/Gerick, Julia/Goldhammer, Frank/Schaumburg, Heike/Schwippert, Knut/Senkbeil, Martin/Vahrenhold, Jan (Hrsg.): ICILS 2018 \#Deutschland. Computer- und informationsbezogene Kompetenzen von Schülerinnen und Schülern im zweiten internationalen Vergleich und Kompetenzen im Bereich Computational Thinking. Münster/New York: Waxmann, S. 205-240.

Eribon, Didier (2017): Rückkehr nach Reims. Berlin: Suhrkamp.

Fend, Helmut (2008): Neue Theorie der Schule. Einführung in das Verstehen von Bildungssystemen. Wiesbaden: Springer VS.

Fickermann, Detlef/Edelstein, Benjamin (2020): „Langsam vermisse ich die Schule...“ - Schule während und nach der Corona-Pandemie. Die Deutsche Schule - Zeitschrift für Erziehungswissenschaft, Bildungspolitik und pädagogische Praxis, Beiheft, 16, Münster/New York: Waxmann.

Fischer, Christian/Fischer-Ontrup, Christiane/Schuster, Corinna (2020): Individuelle Förderung und selbstreguliertes Lernen. Bedingungen und Optionen für das Lehren und Lernen in Präsenz und auf Distanz. In: Fickermann, Detlef/Edelstein, Benjamin (Hrsg.): „Langsam vermisse ich die Schule..." - Schule während und nach der Corona-Pandemie. Die Deutsche Schule - Zeitschrift für Erziehungswissenschaft, Bildungspolitik und pädagogische Praxis, Beiheft 16, Münster/New York: Waxmann, S. 136-152. 
forsa (2020): Die Schule aus Sicht der Schulleiterinnen und Schuleiter vor und während der Corona Krise. Ergebnisse einer repräsentativen Befragung von Schulleitungen in Baden-Württemberg. 5.10.2020. forsa - Politik und Sozialforschung GmbH.

Foucault, Michel (2016): Überwachen und Strafen. Die Geburt des Gefängnisses. Frankfurt am Main: Suhrkamp.

Gogolin, Ingrid (2020): Sprachliche Förderung, sprachliche Bildung und Lernen im Deutschen als Zweitsprache während und nach der Pandemie. In: Fickermann, Detlef/Edelstein, Benjamin (Hrsg.): „Langsam vermisse ich die Schule...“ - Schule während und nach der Corona-Pandemie. Die Deutsche Schule - Zeitschrift für Erziehungswissenschaft, Bildungspolitik und pädagogische Praxis, Beiheft, 16, Münster/New York: Waxmann, S. 175-188.

Goldan, Janka/Geist, Sabine/Lütje-Klose, Birgit (2020): Schüler*innen mit sonderpädagogischem Förderbedarf während der Corona-Pandemie. Herausforderungen und Möglichkeiten der Förderung - Das Beispiel der Laborschule Bielefeld. In: Fickermann, Detlef/Edelstein, Benjamin (Hrsg.): „Langsam vermisse ich die Schule..." - Schule während und nach der Corona-Pandemie. Die Deutsche Schule - Zeitschrift für Erziehungswissenschaft, Bildungspolitik und pädagogische Praxis, Beiheft, 16, Münster/New York: Waxmann, S. 37-60.

Hamborg, Steffen (2018): Lokale Bildungslandschaften auf Nachhaltigkeitskurs. Wiesbaden: Springer VS.

Helm, Christoph/Huber, Stephan/Loisinger, Tina (2021): Was wissen wir über schulische Lehr-LernProzesse im Distanzunterricht während der Corona-Pandemie? - Evidenz aus Deutschland, Österreich und der Schweiz. In: Zeitschrift für Erziehungswissenschaft. URL: doi.org/10.1007/s11618021- 01000-z (27.07.2021).

Helmke, Andreas (2012): Unterrichtsqualität und Lehrerprofessionalität. Seelze: Klett Kallmeyer.

Hoffmann, Ilka (2020): Die Corona-Pandemie als Katalysator für Schulreformen? Ein persönlicher Blick auf die pädagogische Corona-Praxis. In: Fickermann, Detlef/Edelstein, Benjamin (Hrsg.): „Langsam vermisse ich die Schule...“ - Schule während und nach der Corona-Pandemie. Die Deutsche Schule - Zeitschrift für Erziehungswissenschaft, Bildungspolitik und pädagogische Praxis, Beiheft, 16, Münster/New York: Waxmann, S. 95-104.

Huber, Stephan Gerhard/ Günther, Paula Sophie/ Schneider, Nadine/ Helm, Christoph/Schwander, Marius/ Schneider, Julia/ Pruitt, Jane (2020a): COVID-19 - aktuelle Herausforderungen in Schule und Bildung. Erste Befunde des Schul-Barometers in Deutschland, Österreich und der Schweiz. Münster: Waxmann.

Huber, Stephan Gerhard/Helm, Christoph (2020b): Lernen in Zeiten der Corona-Pandemie. Die Rolle familiärer Merkmale für das Lernen von Schüler*innen: Befunde vom Schul-Barometer in Deutschland, Österreich und der Schweiz. In: Fickermann, Detlef/Edelstein, Benjamin (Hrsg.): „Langsam vermisse ich die Schule...“ - Schule während und nach der Corona-Pandemie. Die Deutsche Schule - Zeitschrift für Erziehungswissenschaft, Bildungspolitik und pädagogische Praxis, Beiheft, 16, Münster/New York: Waxmann, S. 37-60.

Jäger, Siegfried (2012): Kritische Diskursanalyse. Eine Einführung. Münster: Unrast.

Kaldewey, David (2014): System, Diskurs, Semantik. Methodologische Überlegungen zum Verhältnis von Differenzierungstheorie, Kultursoziologie und Wissenssoziologie. In: Löw, Martina (Hrsg.): Vielfalt und Zusammenhalt. Verhandlungen des 36. Kongresses der Deutschen Gesellschaft für Soziologie in Bochum und Dortmund 2012. Frankfurt/New York: Campus.

Keller, Reiner (2008): Diskurse und Dispositive analysieren. Die wissenssoziologische Diskursanalyse als Beitrag zu einer wissenschaftlichen Profilierung der Diskursforschung. In: Historical Social Research 33 (1), S. 73-107. 
Keller, Reiner (2011a): Wissenssoziologische Diskursanalyse. Grundlegung eines Forschungsprogramms. Wiesbaden: Springer VS.

Keller, Reiner (2011b): Diskursforschung. Eine Einführung für SozialwissenschaftlerInnen. Wiesbaden: VS Verlag.

Keller, Reiner (2014): Wissenssoziologische Diskursanalyse und Deutungsmusteranalyse. In: Behnke, Cornelia/Lengersdorf, Diana/Scholz, Sylka (Hrsg.): Wissen - Methode - Geschlecht: Erfassen des fraglos Gegebenen. Wiesbaden: Springer VS, S. 143-160.

Kerres, Michael (2003): Wirkungen und Wirksamkeit neuer Medien in der Bildung. In: Keill-Slawik, Reinhard (Hrsg.): Education Quality Forum: Wirkungen und Wirksamkeit neuer Medien. Münster: Waxmann.

Klieme, Eckhard (2020): Guter Unterricht - auch und besonders unter Einschränkungen der Pandemie? In: Fickermann, Detlef/Edelstein, Benjamin (Hrsg.): „Langsam vermisse ich die Schule...“ Schule während und nach der Corona-Pandemie. Die Deutsche Schule - Zeitschrift für Erziehungswissenschaft, Bildungspolitik und pädagogische Praxis, Beiheft, 16, Münster/New York: Waxmann, S. 117-136.

Knaus, Thomas (2018): [Me]nsch - Werkzeug - [I]nteraktion. Theoretisch-konzeptionelle Analysen zur „Digitalen Bildung“ und zur Bedeutung der Medienpädagogik in der nächsten Gesellschaft. In: MedienPädagogik 31, S. 1-35. URL: doi.org/10.21240/mpaed/31/2018.03.26.X (25.07.2021).

Kolbe Fritz-Ulrich/Combe, Arno (2008): Lehrerbildung. In: Helsper Werner/Böhme Jeanette (Hrsg.): Handbuch der Schulforschung. VS Verlag für Sozialwissenschaften, S. 877-904.

Köller, Olaf/Fleckenstein, Johanna/Guill, Karin/Meyer, Jennifer (2020): Pädagogische und dialektische Anforderungen an die häusliche Aufgabenstellung. In: Fickermann, Detlef/Edelstein, Benjamin (Hrsg.): „Langsam vermisse ich die Schule..." - Schule während und nach der Corona-Pandemie. Die Deutsche Schule - Zeitschrift für Erziehungswissenschaft, Bildungspolitik und pädagogische Praxis, Beiheft, 16, Münster/New York: Waxmann, S. 163-174.

Link, Jürgen (2006): Versuch über den Normalismus. Wie Normalität produziert wird. Göttingen: Vandenhoek \& Ruprecht.

Lüders, Christian/Meuser, Michael (1997): Deutungsmusteranalyse. In: Hitzler, Ronald/Honer, Arne (Hrsg.): Sozialwissenschaftliche Hermeneutik. Wiesbaden: Springer VS, S. 57-80.

Luhmann, Niklas (1991): Das Kind als Medium der Erziehung. In: Zeitschrift für Pädagogik 37 (1), S. 19-40.

Macgilchrist, Felicitas (2018): Stirbt das Buch aus und kommt das Tablet? Über die Zukunft des Schulbuches in digitalen Zeiten. Vier Viertel Kult, Frühling/Sommer, S. 8-9.

Maurer, Christian/Rincke, Karsten/Hemmer, Michael (2021): Fachliche Bildung und digitale Transformation - Fachdidaktische Forschung und Diskurse. In: Fachtagung der Gesellschaft für Fachdidaktik. Regensburg: Universität.

McLuhan, Marshall/Fiore, Quentin (2016): Das Medium ist die Message. Stuttgart: Tropen.

Meuser, Michael (2006): Deutungsmusteranalyse. In: Bohnsack, Ralf/Marotzki, Winfried/Meuser, Michael (Hrsg.): Hauptbegriffe Qualitative Sozialforschung. Wiesbaden: Springer VS, S. 21-33.

Meyer, Hilbert (2016): Was ist guter Unterricht? Berlin: Cornelsen.

Muckel, Petra (2011): Die Entwicklung von Kategorien mit der Methode der Grounded Theory. In: Mey, Günther/Mruck, Katja (Hrsg.): Grounded Theory Reader. Wiesbaden: VS Verlag, S. 333-352.

Nassehi, Armin (2019): Muster. Theorie der digitalen Gesellschaft. München: C.H. Beck. 
Niedlich, Sebastian (2020): Neue Ordnung der Bildung. Zur Steuerungslogik der Regionalisierung im deutschen Bildungssystem. Wiesbaden: Springer VS.

Oevermann, Ulrich (1973): Zur Analyse der Struktur von sozialen Deutungsmustern. In: Sozialer Sinn, 1, S. 3-33.

Oevermann, Ulrich (2001): Kommentar zu Christine Plaß und Michael Schetsche. Grundzüge einer wissenssoziologischen Theorie sozialer Deutungsmuster. In: Sozialer Sinn, 3, S. 537-546.

Porsch, Raphaela/Porsch, Thorsten (2020): Fernunterricht als Ausnahmesituation. Befunde einer bundesweiten Befragung von Eltern mit Kindern in der Grundschule. In: Fickermann, Detlef/Edelstein, Benjamin (Hrsg.): „Langsam vermisse ich die Schule...“ - Schule während und nach der Corona-Pandemie. Die Deutsche Schule - Zeitschrift für Erziehungswissenschaft, Bildungspolitik und pädagogische Praxis, Beiheft, 16, Münster/New York: Waxmann, S. 61-78.

Rosa, Harmut (2021): Vorwort. In: August, Vincent (Hrsg.): Technologisches Regieren. Der Aufstieg der Netzwerk-Denkens in der Krise der Moderne. Foucault, Luhmann und die Kybernetik. Bielefeld: Transcript, S. 9.

SAFL (2016): Literaturhinweise Pädagogik. URL: gwhrs.seminar-schwaebisch-gmuend.de/site/pbsbw-km-root/get/documents_E1497549971/KULTUS.Dachmandant/KULTUS/Seminare/seminar-schwaebisch-gmuend-gwhrs/Faecher/GWHS-Paedagogik/Literaturliste\%20neu.pdf (30.07.2021).

Schetsche, Michael/Schmied-Knittel, Ina (2013): Deutungsmuster im Diskurs. Zur Möglichkeit der Integration von Deutungsmustern in die wissenssoziologische Diskursanalyse. In: Zeitschrift für Diskursforschung, 1 (1), S. 24-45.

Schütz, Alfred (1971): Gesammelte Aufsätze I. Das Problem der sozialen Wirklichkeit. Den Haag: Martinus Nijhoff.

Schweiger, Gottfried (2020): Benachteiligte Familien und die ungleiche Last der Pandemie. In: Drerup, Johannes/Schweiger, Gottfried (Hrsg.): Bildung und Erziehung im Ausnahmezustand. Philosophische Reflexionsangebote zur COVID-19 Pandemie. Darmstadt: wbg Academic, S. 108-118.

Sitter, Miriam (2016): PISAs fremde Kinder. Eine diskursanalytische Studie. Wiesbaden: Springer VS.

Strauss, Anselm/Corbin, Juliet (1996): Grounded theory. Grundlagen qualitativer Sozialforschung. Weinheim: Beltz.

Tenorth, Heinz-Elmar (2020): Voll digital in die pädagogische Regression. In: Das Deutsche Schulportal. URL: deutsches-schulportal.de/stimmen/voll-digital-in-die-paedagogische-regression/ (18.04.2021).

Ullrich, Carsten (1999): Deutungsmusteranalyse und diskursives Interview. In: Zeitschrift für Soziologie, 28, Heft (6), S. 429-447.

Ullrich, Carsten (2020): Das Diskursive Interview. Methodische und methodologische Grundlagen. Wiesbaden: Springer VS.

VBE (2021): Hinsehen! Corona-Unmut bedroht Schulfrieden. URL: vbe.de/presse/pressedienste/pressedienste-2021/hinsehen-corona-unmut-bedroht-schulfrieden (11.05.2021).

Wacker, Albrecht/Unger, Valentin/Rey, Thomas (2020): „Sind doch Corona-Ferien, oder nicht?“. Befunde einer Schüler*innenbefragung zum Fernunterricht. In: Fickermann, Detlef/Edelstein, Benjamin (Hrsg.): „Langsam vermisse ich die Schule...“ - Schule während und nach der CoronaPandemie. Die Deutsche Schule - Zeitschrift für Erziehungswissenschaft, Bildungspolitik und pädagogische Praxis, Beiheft, 16, Münster/New York: Waxmann, S. 79-94. 
Wrase, Michael (2020): Schulrechtliche Herausforderungen in Zeiten der Corona-Pandemie. In: Fickermann, Detlef/Edelstein, Benjamin (Hrsg.): „Langsam vermisse ich die Schule...“ - Schule während und nach der Corona-Pandemie. Die Deutsche Schule - Zeitschrift für Erziehungswissenschaft, Bildungspolitik und pädagogische Praxis, Beiheft, 16, Münster/New York: Waxmann, S. 105-116.

\section{Informationen zum Autor}

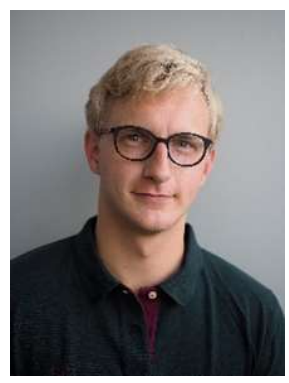

Carlo Schmidt ist Realschullehrer und Lehrbeauftragter am Institut für Erziehungswissenschaften/Abteilung Allgemeine Pädagogik an der Pädagogischen Hochschule Ludwigsburg. Seine Forschungsschwerpunkte sind zur Zeit: erziehungswissenschaftliche Diskurs- und Deutungsmusteranalysen sowie systemtheoretische Beobachtungen erziehungswissenschaftlichen Wissens.

carlo-leonard.schmidt@gmx.de

Zitationshinweis:

Schmidt, Carlo (2021): Disruptionen in der Schule aus der Sicht von Lehrer*innen. In: Online-Magazin Ludwigsburger Beiträge zur Medienpädagogik, Ausgabe 21/2021. URL: medienpaed-ludwigsburg.de/ 